\title{
Hepatitis B Virus Reactivation Following Salazosulfapyridine Monotherapy in a Patient with Rheumatoid Arthritis
}

\author{
Kengo Akashi ${ }^{1}$, Jun Saegusa ${ }^{1}$, Yuji Nakamachi ${ }^{2}$, Takashi Nakazawa ${ }^{3}$, \\ Shunichi Kumagai ${ }^{4}$ and Akio Morinobu ${ }^{1}$
}

\begin{abstract}
A 72-year-old man was diagnosed with rheumatoid arthritis (RA) and prior hepatitis B virus (HBV) infection. He began treatment with salazosulfapyridine (SASP). Several months later, his blood tests reflected a slightly elevated liver function. Serum tests were positive for hepatitis B surface antigen and HBV-DNA, and the diagnosis of de novo HBV hepatitis was made. A genetic analysis showed that he had polymorphisms of $A B C G 2$ and NAT2, which could lead to high plasma concentrations of SASP and sulfapyridine. To the best of our knowledge, this is the first report of de novo hepatitis developing during SASP monotherapy for RA.
\end{abstract}

Key words: rheumatoid arthritis, hepatitis B virus, de novo HBV hepatitis, salazosulfapyridine

(Intern Med 55: 1371-1373, 2016)

(DOI: 10.2169/internalmedicine.55.5425)

\section{Introduction}

Hepatitis B virus (HBV) can become reactivated in patients with rheumatic diseases who are treated with intensive immunosuppressive therapy with or without biological disease-modifying antirheumatic drugs (DMARDs). De novo HBV hepatitis, in which patients with reactivated HBV are negative for hepatitis $B$ surface antigen (HBsAg), positive for hepatitis B surface antibody (HBsAb) and hepatitis B core antibody ( $\mathrm{HBcAb}$ ), and negative for HBV-DNA, is particularly important because it has a high mortality rate and is likely to be fulminant.

Salazosulfapyridine (SASP) is an immunomodulatory DMARD that is widely used for rheumatoid arthritis (RA). SASP treatment has received little attention in regard to HBV management in RA patients (1) because there have been no reports of de novo HBV hepatitis induced by SASP. ATP-binding cassette protein G2 (ABCG2) and Nacetyltransferase 2 (NAT2) are associated with the absorption and metabolism of SASP. The ABCG2 and NAT2 genes exhibit hereditarily determined polymorphisms. It has been reported that these polymorphisms are related to the efficacy and toxicity of SASP. We herein report a case of de novo hepatitis that developed in a man with RA during SASP monotherapy who had specific polymorphisms of $A B C G 2$ and NAT2.

\section{Case Report}

The patient was a 72-year-old man diagnosed with RA in December 2011; he presented with symmetrical small-joint arthritis demonstrated by musculoskeletal ultrasonography and was positive for anti-citrullinated protein antibody (16.3 $\mathrm{U} / \mathrm{mL})$ and an abnormal C-reactive protein level $(0.7 \mathrm{mg} /$ dL). Serological findings suggested a previous HBV infection; the patient was negative for $\mathrm{HBsAg}$ and serum HBVDNA and positive for HBsAb and $\mathrm{HBcAb}$. He began treatment with SASP (250 mg twice daily), and RA promptly went into clinical remission. He remained on SASP monotherapy thereafter. In March 2013, when the patient was 74 years of age, his laboratory tests showed a slightly elevated

\footnotetext{
${ }^{1}$ Department of Rheumatology and Clinical Immunology, Kobe University Graduate School of Medicine, Japan, ${ }^{2}$ Department of Clinical Laboratory, Kobe University Hospital, Japan, ${ }^{3}$ Department of Rheumatology, Osaka Saiseikai Nakatsu Hospital, Japan and ${ }^{4}$ The Center for Rheumatic Diseases, Shinko Hospital, Japan

Received for publication March 24, 2015; Accepted for publication August 16, 2015

Correspondence to Dr. Jun Saegusa, jsaegusa@med.kobe-u.ac.jp
} 


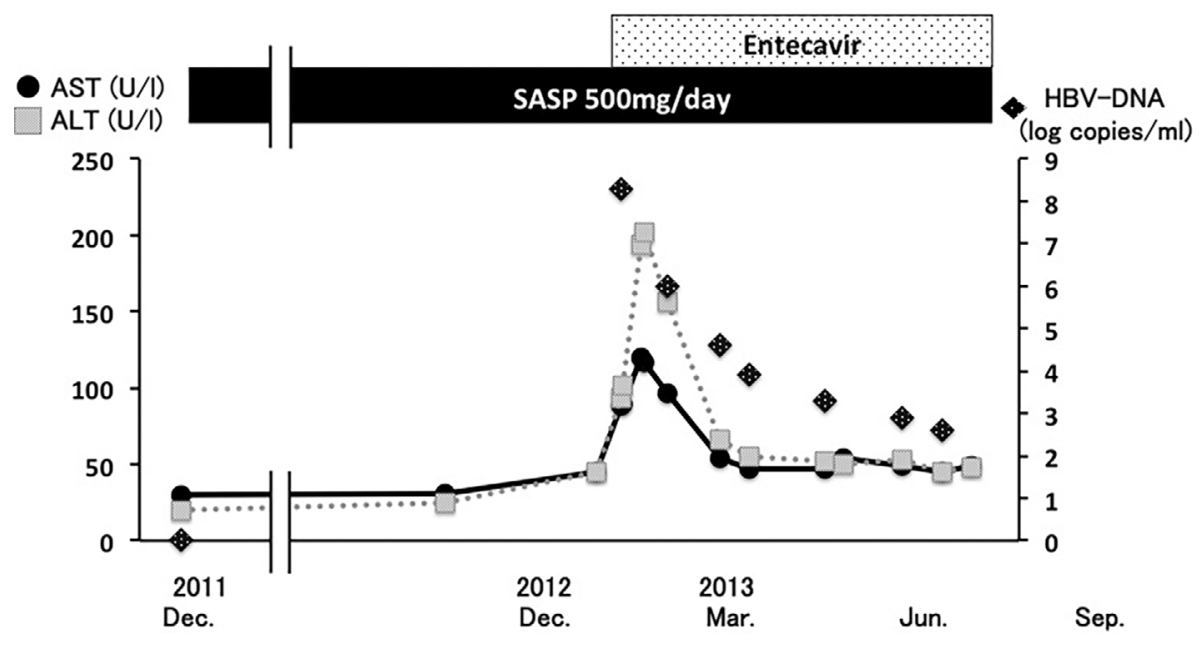

Figure. The development of de novo hepatitis during SASP treatment. The AST, ALT and HBVDNA levels are shown. AST: aspartate transaminase, ALT: alanine transaminase, SASP: salazosulfapyridine, HBV-DNA: hepatitis B virus DNA

liver function (AST: $88 \mathrm{U} / \mathrm{L} ; \mathrm{ALT}: 94 \mathrm{U} / \mathrm{L}$ ). The serum tests were positive for HBsAg, and serum HBV-DNA was $8.3 \mathrm{log}$ copies/mL (normal range: $<2.1 \log$ copies/mL). Other viral infections such as cytomegalovirus, Epstein-Barr virus, herpes simplex virus, varicella zoster virus, hepatitis A virus, and hepatitis $\mathrm{C}$ virus were ruled out through laboratory tests. The diagnosis was de novo $\mathrm{HBV}$ hepatitis potentially associated with SASP monotherapy, although it appeared that the patient was not in a severe immunosuppressive state because his laboratory test showed a slight decrease in the WBC and IgG concentration at the onset of hepatitis; WBC:

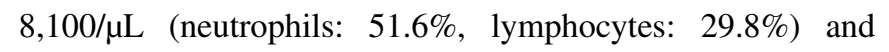
IgG: $1,819 \mathrm{mg} / \mathrm{dL}$ at the diagnosis of RA, and WBC: $5,500 /$ $\mu \mathrm{L}$ (neutrophils: 63.0\%, lymphocytes: 21.0\%) and IgG: $1,618 \mathrm{mg} / \mathrm{dL}$ at the onset of de novo HBV hepatitis. The patient continued SASP and began entecavir treatment, and the liver function test values and serum HBV viral load gradually returned to a normal range (Figure). After obtaining informed consent from the patient, we examined his NAT2 and $A B C G 2$ genotypes; he was found to have $N A T 2 * 6 A / 6 A$, which is classified as a "slow acetylator genotype," and $A B C G 2-A / A$, resulting in high plasma concentrations of SASP.

\section{Discussion}

To the best of our knowledge, this is the first report of de novo $\mathrm{HBV}$ hepatitis occurring in a patient with RA during SASP monotherapy. A recent case-control study of the associations between hepatitis $B$ and DMARD therapy in RA patients reported 98,161 adverse events (AEs) associated with biologic and conventional synthetic DMARDs, including $92 \mathrm{HBV}$-associated AEs. There were 1,340 AEs in SASP-treated patients, including four $\mathrm{HBV}$-associated AEs (2). In each of these four cases, concomitant immunosuppressive agents, such as methotrexate, biologic DMARDs, and glucocorticoids, had been used and the pa- tients had no pattern of previous HBV infection (3).

SASP is constructed of 5-aminosalicylic acid (5-ASA) linked by an azo bond to sulfapyridine (SP). When administered orally, some SASP is absorbed in the gastrointestinal tract via $\mathrm{ABCG} 2$, an efflux $\mathrm{ABC}$ transporter that is expressed at the apical membrane of enterocytes. The remaining SASP is split into 5-ASA and SP by bacterial action in the colon; 5-ASA remains largely within the large bowel, while SP is completely absorbed and metabolized to $\mathrm{N}$ acetylsulfapyridine (AcSP), predominantly by NAT2. As mentioned above, the $A B C G 2$ and NAT2 genes exhibit hereditarily determined polymorphisms. The human NAT2 phenotypes can be classified as rapid, intermediate, or slow acetylators. Since slow acetylators typically exhibit a high plasma concentration of SP and a low plasma concentration of AcSP, SASP is both more effective and more toxic for slow acetylators than for the other phenotypes (4). Similar variants have been identified in the $A B C G 2$ gene. Among them, the 421C>A (141Gln>Lys in exon 5) genotype is important; plasma SASP concentrations are significantly higher in $A B C G 2-\mathrm{A} / \mathrm{A}$ subjects than in $\mathrm{C} / \mathrm{C}$ and $\mathrm{C} / \mathrm{A}$ subjects (5). $A B C G 2$ polymorphisms are also associated with SASP efficacy (6). Thus higher concentrations of SASP and SP appear to be related to a higher efficacy and risk of AEs. We did not check the plasma concentrations of SASP and SP in our patient. However, we speculate that his concentrations of SASP and SP were high because he had the $A B C G 2-A / A$ and $N A T 2 * 6 A / * A$ polymorphisms.

SASP is reported to suppress T-cell activation and proliferation by inhibiting the nuclear factor kappa B (NFKB) signaling pathway (7). SASP and SP are also reported to suppress $\operatorname{IgM}$ and $\operatorname{IgG}$ production by $\mathrm{B}$ cells (8). Thus, high plasma concentrations of SASP and SP could suppress the immune system, resulting in HBV reactivation. However, the patient's lymphocytopenia and hypogammaglobulinemia were not severe to the extent that they caused de novo HBV hepatitis themselves. As the mechanisms of SASP's anti- 
rheumatic action are not completely understood, other immunosuppressive mechanisms of SASP might be associated with de novo HBV hepatitis in the present case.

It has been reported that the mutation of the HBV gene is associated with $\mathrm{HBV}$ reactivation following chemotherapy. For instance, a point mutation at position A1896 of the precore region $(9,10)$ and dinucleotide mutation (T1762/ A1764) in the basic core promoter region (11) have been described to be related to de novo $\mathrm{HBV}$ hepatitis development in patients who received cytotoxic chemotherapy for cancer. Unfortunately, whether or not these mutations are involved in the present patient's de novo hepatitis remains unknown because we did not check for these mutations.

Our patient developed de novo HBV hepatitis while being treated with only $250 \mathrm{mg}$ SASP taken twice daily. Although de novo $\mathrm{HBV}$ hepatitis may result from various contributing factors, immunosuppression due to high plasma concentrations of SASP and SP was assumed to be associated with $\mathrm{HBV}$ reactivation in the present case. To the best of our knowledge, this is the first report of de novo hepatitis developing during SASP monotherapy for RA. While this is a rare case, RA patients with a history of $\mathrm{HBV}$ infection should be managed carefully, even when treated with SASP alone.

The authors state that they have no Conflict of Interest (COI).

\section{References}

1. Calabrese LH, Zein NN, Vassilopoulos D. Hepatitis B virus (HBV) reactivation with immunosuppressive therapy in rheumatic diseases: assessment and preventive strategies. Ann Rheum Dis 65: 983-989, 2006.
2. Oshima Y, Tsukamoto H, Tojo A. Association of hepatitis B with antirheumatic drugs: a case-control study. Mod Rheumatol 23: 694-704, 2013.

3. Zingarelli S, Airò P, Frassi M, Bazzani C, Scarsi M, Puoti M. Prophylaxis and therapy of HBV infection in 20 patients treated with disease modifying antirheumatic drugs or with biological agents for rheumatic diseases. Reumatismo 60: 22-27, 2008.

4. Kumagai S, Komada F, Kita $T$, et al. N-acetyltransferase 2 genotype-related efficacy of sulfasalazine in patients with rheumatoid arthritis. Pharm Res 21: 324-329, 2004.

5. Yamasaki Y, Ieiri I, Kusuhara H, et al. Pharmacogenetic characterization of sulfasalazine disposition based on NAT2 and ABCG2 (BCRP) gene polymorphisms in humans. Clin Pharmacol Ther 84: 95-103, 2008.

6. Wiese MD, Alotaibi N, O'Doherty C, et al. Pharmacogenomics of NAT2 and ABCG2 influence the toxicity and efficacy of sulphasalazine containing DMARD regimens in early rheumatoid arthritis. Pharmacogenomics J 14: 350-355, 2014.

7. Liptay S, Bachem M, Häcker G, Adler G, Debatin KM, Schmid RM. Inhibition of nucleolar factor kappa $\mathrm{B}$ and induction of apoptosis in T-lymphocytes by sulfasalazine. Br $\mathrm{J}$ Pharmacol 128: 1361-1369, 1999.

8. Hirohata S, Ohshima N, Yanagida T, Aramaki K. Regulation of human B cell function by sulfasalazine and its metabolites. Int Immunopharmacol 2: 631-640, 2002.

9. Chen PM, Yao NS, Wu CM, et al. Detection of reactivation and genetic mutations of the hepatitis B virus in patients with chronic hepatitis B infections receiving hematopoietic stem cell transplantation. Transplantation 74: 182-188, 2002.

10. Steinberg JL, Yeo W, Zhong S, et al. Hepatitis B virus reactivation in patients undergoing cytotoxic chemotherapy for solid tumours: precore/core mutations may play an important role. J Med Virol 60: 249-255, 2000.

11. Lau GK, Leung YH, Fong DY, et al. High hepatitis B virus (HBV) DNA viral load as the most important risk factor for HBV reactivation in patients positive for HBV surface antigen undergoing autologous hematopoietic cell transplantation. Blood 99: 23242330, 2002.

\footnotetext{
(C) 2016 The Japanese Society of Internal Medicine http://www.naika.or.jp/imonline/index.html
} 